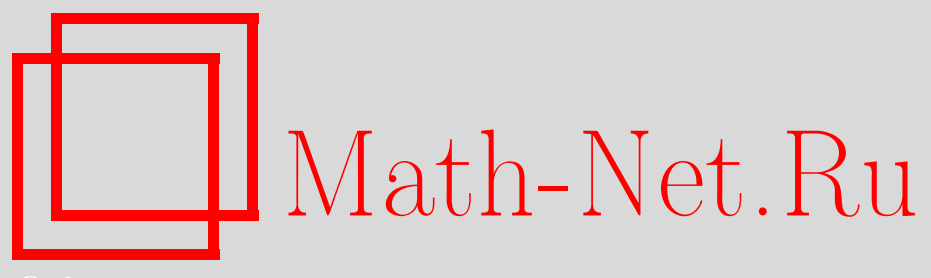

С. В. Бородачов, Э. Б. Сафф, Д. П. Хардин, Асимптотика плотнейших взвешенных упаковок на спрямляемых множествах, Матем. сб., 2008, том 199, номер 11, 3-20

DOI: https://doi.org/10.4213/sm3943

Использование Общероссийского математического портала Math-Net.Ru подразумевает, что вы прочитали и согласны с пользовательским соглашением http: //www. mathnet.ru/rus/agreement

Параметры загрузки:

IP: 107.22 .136 .117

26 апреля 2023 г., 11:44:17

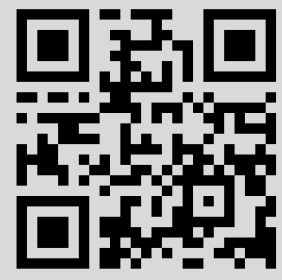




\author{
С. В. Бородачов, Э. Б. Сафф, Д. П. Хардин
}

\title{
Асимптотика плотнейших взвешенных упаковок на спрямляемых множествах
}

\begin{abstract}
Исследуется асимптотическое поведение с ростом $N$ наибольшего значения минимума взвешенных расстояний между парами точек из системы $N$ точек спрямляемого компакта в евклидовом пространстве. Находится предельное распределение асимптотически оптимальных конфигураций.

Библиография: 23 названия.
\end{abstract}

Классической задачей о плотных упаковках является поиск конфигурации $N$ "максимально разделенных" точек фиксированного компакта $A$ (доставляющих наибольший возможный минимум расстояний между парами точек). Поставленная для $\mathbb{R}^{d}$, она становится задачей о наибольшей плотности равных неналегающих шаров в $\mathbb{R}^{d}$. Ряд важных результатов и обзоры литературы по этой задаче можно найти в [1]-[6]. K примеру, известно, что для некоторых плоских множеств $A$ решение этой задачи дается асимптотически оптимальными узлами кубатурных формул (см., например, [7]).

Задача о плотной упаковке двойственна задаче о $\varepsilon$-сложности компакта $A$, в которой при фиксированном $\varepsilon>0$ требуется найти максимальное число точек в $A$, лежащих на расстоянии не меньше $\varepsilon$ друг от друга. Понятие $\varepsilon$-сложности было первоначально введено А.Н. Колмогоровым и В. М. Тихомировым [8] и имеет, например, приложения к исследованию сложности поведения орбит динамических систем (см., например, [9]).

Вышеупомянутые задачи рассматривались и в других метрических пространствах. В настоящей работе мы изучаем взвешенный вариант задачи о плотнейшей упаковке, который в известном смысле аналогичен введению некоторой метрики или последовательности метрик. Решение этой задачи может быть использовано в конструкции оптимальных взвешенных кубатурных формул (см., к примеру, [10]) и в компьютерном геометрическом моделировании, например, когда требуется расположить точки на поверхности в соответствии с заданным неравномерным распределением (скажем, так, чтобы точки плотнее заполняли области большей кривизны на поверхности).

\section{§1. Определения, обозначения и известные результаты}

Пусть $A$ - бесконечное подмножество $\mathbb{R}^{p}$, где $p$ - натуральное число. Для набора различных точек $\omega_{N}:=\left\{x_{1}, \ldots, x_{N}\right\} \subset A$ и функции $w: A \times A \rightarrow[0, \infty]$

Исследования были частично поддержаны NSF (у первого автора - гранты №№ DMS0505756, DMS-0532154, у второго автора - гранты №№ DMS-0532154, DMS-0603828, у третьего автора - гранты №№ DMS-0603828, DMS-0532154.)

(С) С. В. БородАчов, Э.Б. САФФ, Д. П. ХАРДиН, 2008 
положим

$$
\delta^{w}\left(\omega_{N}\right):=\min _{1 \leqslant i \neq j \leqslant N} w\left(x_{i}, x_{j}\right)\left|x_{i}-x_{j}\right|,
$$

где $|\cdot|$ - евклидово расстояние в $\mathbb{R}^{p}$. Также введем расстояние плотнейшей взвешенной $N$-точечной упаковки на $A$

$$
\delta_{N}^{w}(A):=\sup \left\{\delta^{w}\left(\omega_{N}\right): \omega_{N} \subset A, \# \omega_{N}=N\right\}
$$

где $\# X-$ мощность множества $X$. Нас интересует асимптотика (при $N \rightarrow \infty$ ) величин (1), а также асимптотика конфигураций, доставляющих верхнюю грань правой части (1).

Мы можем считать без ограничения общности, что $w$ - симметричная функция, так как при $v(x, y):=\min \{w(x, y), w(y, x)\}$ имеем $\delta^{v}\left(\omega_{N}\right)=\delta^{w}\left(\omega_{N}\right)$ для всякой конфигурации $\omega_{N}$.

При отсутствии веса $(w \equiv 1)$ мы получаем классическую задачу о наилучшей упаковке (при $w \equiv 1$ опускаем верхний индекс $w$ в определении (1) и вообще всюду). В этом случае (возможно, неединственные) конфигурации $\omega_{N}$, доставляющие верхнюю грань в (1), называются конфигурациями плотнейшей упаковки в $A$. Заметим, что в случае, когда $A$ - сфера $S^{2}$ в $\mathbb{R}^{3}$, точное решение задачи о плотнейшей упаковке было получено при $N=2, \ldots, 12$ и $N=24$ (см. ссылки в [4] или [5]). Асимптотическое поведение при $N \rightarrow \infty$ расстояния плотнейшей упаковки на $S^{2}$ (с точностью до следующего по порядку члена) было найдено Хабихтом и ван дер Варденом в [11] и [12].

В работе [13] авторы проанализировали асимптотическое поведение расстояния плотнейшей упаковки в невзвешенном случае для $d$-спрямляемых множеств и их счетных объединений.

В настоящей статье мы обозначаем через $\mathscr{H}_{\alpha}$, где $\alpha \leqslant p$, хаусдорфову $\alpha$-меру в $\mathbb{R}^{p}$. Если $\alpha=d$ - целое число, мы выберем нормализацию, при которой изо-

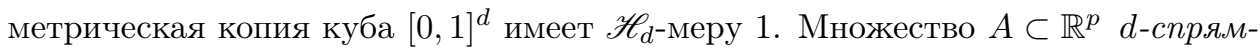
ляемое для $d \leqslant p$, если оно является образом ограниченного подмножества $T$ пространства $\mathbb{R}^{d}$ относительно липшицева отображения, т.е. относительно отображения $\varphi: T \rightarrow \mathbb{R}^{p}$, которое для некоторой постоянной $\lambda$ удовлетворяет неравенству

$$
|\varphi(x)-\varphi(y)| \leqslant \lambda|x-y|, \quad x, y \in T
$$

(см. [14], [15]).

В дальнейшем будем считать, что $A$ - компакт. В первой части статьи мы рассмотрим задачу взвешенной плотнейшей упаковки для фиксированного веса на $A \times A$, удовлетворяющего следующим условиям. Будем называть $w: A \times A \rightarrow[0, \infty]$ CBD-весом на $A \times A$, если:

(a) $w$ непрерывен (как функция на $A \times A$ ) в $\mathscr{H}_{d}$-почти всякой точке диагонали $D(A):=\{(x, x): x \in A\}$;

(b) существует окрестность $G($ в $A \times A)$ диагонали $D(A)$, где $\sup _{G} w<\infty$;

(c) $\inf _{B} w>0$ на всяком замкнутом подмножестве $B$ произведения $A \times A$, удовлетворяющем условию $B \cap D(A)=\varnothing$. 
Мы используем обозначение CBD, чтобы подчеркнуть непрерывность и ограниченность на диагонали. В частности, условия (а)-(с) выполнены при $w$, превосходящем на $A \times A$ положительную константу и непрерывном на диагонали $D(A)$ (где непрерывность в точке диагонали $\left(x_{0}, x_{0}\right)$ подразумевается в смысле пределов на $A \times A)$.

Заметим, что $w(x, y)|x-y|$, вообще говоря, не метрика, однако если метрика $\rho(x, y)$ непрерывна относительно евклидовой метрики на $\mathbb{R}^{p}$ и предел

$$
f(z):=\lim _{(x, y) \rightarrow(z, z)} \frac{\rho(x, y)}{|x-y|}, \quad(x, y) \in(A \times A) \backslash D(A), \quad z \in A,
$$

существует и непрерывен, то мы получим результаты о плотнейших упаковках в метрике $\rho(x, y)$, полагая

$$
w(x, y):= \begin{cases}\rho(x, y) /|x-y| & \text { при } x \neq y, \\ f(z) & \text { при } x=y=z .\end{cases}
$$

Обзор результатов по упаковкам в неевклидовых пространствах см., например, в [16], [17].

В настоящей работе найдено асимптотическое поведение величины (1), а также *-слабое предельное распределение асимптотически экстремальных конфигураций для $d$-спрямляемых компактов. Затем мы обобщаем эти результаты в двух направлениях, рассматривая переменные веса и допуская наличие особенностей у веса. Если вес может принимать большие значения, то это может существенно повлиять на расстояния между оптимальными точками, что приводит к существенным техническим сложностям для весов с особенностями.

Положим

$$
C_{\infty, d}:=\lim _{N \rightarrow \infty} \delta_{N}\left([0,1]^{d}\right) N^{1 / d} .
$$

Пусть $-\Delta_{d}$ наибольшая плотность упаковки из равных неналегающих шаров в $\mathbb{R}^{d}$ (точное определение см. в [3]), и пусть $\beta_{d}$ - мера Лебега единичного шара в $\mathbb{R}^{d}$. В силу определения

$$
C_{\infty, d}=2\left(\frac{\Delta_{d}}{\beta_{d}}\right)^{1 / d}
$$

Плотность $\Delta_{d}$ (и, соответственно, константа $\left.C_{\infty, d}\right)$ вычислена только при $d=2$ (cp. [1]), а также недавно - при $d=3$ (ср. [6]). Эти результаты показывают, что

$$
C_{\infty, 2}=\sqrt{\frac{2}{\sqrt{3}}}, \quad C_{\infty, 3}=\sqrt[6]{2}
$$

(ясно, что $\left.C_{\infty, 1}=1\right)$.

Задача о плотнейшей упаковке - предельный случай при $s \rightarrow \infty$ следующей задачи о минимуме энергии:

$$
\mathscr{E}_{s}^{w}(A, N)=\inf _{x_{1}, \ldots, x_{N} \in A} \sum_{1 \leqslant i \neq j \leqslant N} \frac{w\left(x_{i}, x_{j}\right)}{\left|x_{i}-x_{j}\right|^{s}} .
$$


Мы рассмотрели эту задачу в [18] для класса весов $w$, включающего в себя функции, обратные (вида $1 / f)$ к CBD-весам, а также более общие веса с конечным числом нулей на диагонали. Мы показали, что если $A \subset \mathbb{R}^{p}$ - замкнутое $d$-спрямляемое множество и $s>d$, то

$$
\lim _{N \rightarrow \infty} \frac{\mathscr{E}_{s}^{w}(A, N)}{N^{1+s / d}}=C_{s, d}\left(\int_{A}(w(x, x))^{-d / s} d \mathscr{H}_{d}(x)\right)^{-s / d},
$$

где $C_{s, d}$ - положительная константа, не зависящая от $A$. При $w \equiv 1$ мы получаем (безвесовую) задачу о минимуме энергии (см. [19]-[23] и ссылки в этих работах).

Предельное распределение асимптотически оптимальных конфигураций понимается в следующем смысле. Если $A \subset \mathbb{R}^{p}-$ компакт и если $\nu$ и $\left\{\nu_{N}\right\}_{N=1}^{\infty}$ суть вероятностные борелевские меры на $A$, то говорится, что последователь-

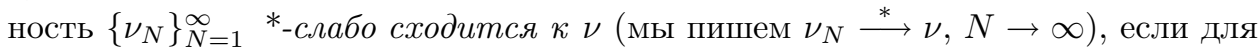
всякой непрерывной функции $f$ на $A$

$$
\lim _{N \rightarrow \infty} \int_{A} f d \nu_{N}=\int_{A} f d \nu
$$

Будем говорить, что последовательность конфигураций $\left\{\omega_{N}\right\}_{N=2}^{\infty}, \omega_{N}=\left\{x_{1, N}\right.$, $\left.\ldots, x_{N, N}\right\} \subset A, N=2,3, \ldots$, имеет предельную вероятностную меру $\nu$, если

$$
\nu\left(\omega_{N}\right):=\frac{1}{N} \sum_{k=1}^{N} \delta_{x_{k, N}} \stackrel{*}{\longrightarrow} \nu, \quad N \rightarrow \infty,
$$

где $\delta_{x}$ - точечная вероятностная мера на $\mathbb{R}^{p}$ в точке $x \in \mathbb{R}^{p}$.

Для доказательства (5) достаточно показать, что для всякого $B \subset A$, относительная граница которого в $A$ имеет $\nu$-меру нуль,

$$
\lim _{N \rightarrow \infty} \frac{\#\left(\omega_{N} \cap B\right)}{N}=\nu(B) .
$$

В статьях [22] и [23] была доказана равномерность предельного распределения конфигураций минимальной $s$-энергии на $d$-спрямляемых подмногообразиях $\mathbb{R}^{p}$ при $s \geqslant d$. В [18] авторы показали, что плотность предельного распределения конфигураций, асимптотически минимизирующих взвешенную энергию в $(4)$, пропорциональна $(w(x, x))^{-d / s}, s>d$. В. Афраймович и Л. Глебский (см. [9]) изучали свойства предельного распределения (в смысле сходимости по ультрафильтру) конфигураций оптимальной $\varepsilon$-сложности на компактных подмножествах $\mathbb{R}^{d}$ с переменной метрикой.

Нашей первой целью будет весовой аналог следующего результата из [13].

Теорема 1.1. Пусть $d u p$ - целье числа, $d \leqslant p, u$ пусть $A \subset \mathbb{R}^{p}$ - бесконечное замкнутое d-спрямляемое множество. Тогда

$$
\lim _{N \rightarrow \infty} \delta_{N}(A) N^{1 / d}=C_{\infty, d} \mathscr{H}_{d}(A)^{1 / d}=2\left(\frac{\Delta_{d}}{\beta_{d}}\right)^{1 / d} \mathscr{H}_{d}(A)^{1 / d} .
$$


При $\mathscr{H}_{d}(A)>0$ для всякой последовательности $\left\{\bar{\omega}_{N}\right\}_{N=2}^{\infty}$ конфигураций плотнейшей упаковки на $А$ такой, что $\# \bar{\omega}_{N}=N, N=2,3, \ldots$, выполнено

$$
\nu\left(\bar{\omega}_{N}\right) \stackrel{*}{\longrightarrow} \frac{\left.\mathscr{H}_{d}\right|_{A}(\cdot)}{\mathscr{H}_{d}(A)}, \quad N \rightarrow \infty .
$$

\section{§ 2. Основные результаты}

Пусть $\mathscr{H}_{d}^{w}$ и $h_{d}^{w}-$ следующие борелевские меры с носителем в $A$ :

$$
\mathscr{H}_{d}^{w}(B)=\int_{B} w(x, x)^{d} d \mathscr{H}_{d}(x)
$$

и

$$
h_{d}^{w}(B)=\frac{\mathscr{H}_{d}^{w}(B)}{\mathscr{H}_{d}^{w}(A)}
$$

для всякого борелевского множества $B \subset A$. Будем говорить, что последовательность $\left\{\omega_{N}\right\}_{N=2}^{\infty}, \# \omega_{N}=N, N=2,3, \ldots$, конфигураций в $A$ реализует асимптотически $w$-плотнейшую упаковку на $A$, если

$$
\delta^{w}\left(\omega_{N}\right)=\delta_{N}^{w}(A)(1+o(1)), \quad N \rightarrow \infty .
$$

Теорема 2.1. Пусть $d \leqslant p$ для иелых $d$ u $p$. Пусть $A \subset \mathbb{R}^{p}$ - замкнутое бесконечное $d$-спрямляемое множество, и пусть $w$ - CBD-весовая функиия на $A \times A$. Тогда

$$
\lim _{N \rightarrow \infty} \delta_{N}^{w}(A) N^{1 / d}=C_{\infty, d}\left(\int_{A} w(x, x)^{d} d \mathscr{H}_{d}(x)\right)^{1 / d},
$$

где $C_{\infty, d}$ та же, что ив (3).

Далее, если $\mathscr{H}_{d}(A)>0$, то для всякой последовательности конфигураций на $A$, асимптотически реализующей $w$-плотнейшую упаковку $\widetilde{\omega}_{N}=\left\{x_{1}^{N}\right.$, $\left.\ldots, x_{N}^{N}\right\}, N=2,3, \ldots$, выполняется

$$
\frac{1}{N} \sum_{k=1}^{N} \delta_{x_{k}^{N}} \stackrel{*}{\longrightarrow} h_{d}^{w}, \quad N \rightarrow \infty .
$$

Пример 2.2. Пусть $\mathbb{D}$ - открытый единичный диск в комплексной плоскости $\mathbb{C}$, и пусть $A$ - бесконечное замкнутое 1-спрямляемое подмножество в $\mathbb{D}$ (к примеру, спрямляемая дуга или кривая) положительной длины (т.е. $\left.\mathscr{H}_{1}(A)>0\right)$. Допустим, что $\omega_{N}^{*}=\left\{z_{1, N}^{*}, \ldots, z_{N, N}^{*}\right\}$ максимизирует минимальное псевдогиперболическое расстояние $d(z, \zeta)=|(z-\zeta) /(1-z \bar{\zeta})|$ среди всех $N$-точечных подмножеств. Тогда из теоремы 2.1 с весом $w(z, \zeta)=1 /|1-z \bar{\zeta}|$, $z, \zeta \in \mathbb{D}$, следует, что точки $\omega_{N}^{*}$ асимптотически равномерно распределены относительно инфинитезимальной метрики Бергмана (Пуанкаре) $|d z| /\left(1-|z|^{2}\right)$.

Аналогично, если $A$ - замкнутое подмножество $D$ положительной площади (т.е. $\mathscr{H}_{2}(A)>0$ ), то для того же веса, что и раньше, конфигурации, реализующие $w$-плотнейшую упаковку, имеют асимптотическую плотность $1 /\left(1-|z|^{2}\right)^{2}$ относительно плоской меры (т.е. $\left.\mathscr{H}_{2}\right)$. 
В теореме 2.1 рассматриваются ограниченные веса на $D(A)$. Ниже мы обсуждаем случай, когда вес может иметь особенности на $D(A)$. Пусть $B(a, r)-$ открытый шар в $\mathbb{R}^{p}$ радиуса $r>0$ с центром в точке $a$. Для $t>0$ скажем, что у функции $w: A \times A \rightarrow[0, \infty]$ имеется особенность в $(a, a) \in D(A)$ порядка не выше $t$, если существуют положительные постоянные $C$ и $\delta$, для которых

$$
w(x, y) \leqslant \frac{C}{|x-a|^{t}}, \quad x, y \in A \cap B(a, \delta) .
$$

Если у $w$ есть особенность $(a, a) \in D(A)$ слишком высокого порядка, то она может играть роль притягивающего стока для оптимальных конфигураций, и в результате $\delta_{N}^{w}(A)=\infty$. K примеру, пусть $A$ - замкнутый шар в $\mathbb{R}^{d}$ с центром в начале координат, и пусть $w(x, y)=|x|^{-t}+|y|^{-t}, x, y \in A, t>1$. Если $\omega_{N}=$ $\left\{x_{1}, \ldots, x_{N}\right\}$ - конфигурация $N$ различных точек $A$, то $\delta^{w}\left(\gamma \omega_{N}\right)=\gamma^{1-t} \delta^{w}\left(\omega_{N}\right)$ для всякого $0<\gamma<1$. Положив $\gamma \rightarrow 0$, мы видим, что $\delta_{N}^{w}(A)=\infty$.

Говорят, что замкнутое подмножество $A \subset \mathbb{R}^{p} \alpha$-регулярно в точке $a \in A$, если существуют такие положительные постоянные $C_{0}$ и $\delta_{0}$, что

$$
C_{0}^{-1} r^{\alpha} \leqslant \mathscr{H}_{\alpha}(A \cap B(x, r)) \leqslant C_{0} r^{\alpha}
$$

для всех $x \in A \cap B\left(a, \delta_{0}\right)$ и $0<r<\delta_{0}$.

ТеОрема 2.3. Пусть $A \subset \mathbb{R}^{p}$ - бесконечное замкнутое $d$-спрямляемое множество $d \leqslant p$. Для конечного набора точек $a_{1}, \ldots, a_{n} \in A$ допустим, что $A$ $\alpha_{i}$-регулярно в $a_{i}$ с $\alpha_{i} \leqslant d, i=1, \ldots, n$, и пусть $w: A \times A \rightarrow[0, \infty]$ - CBD-весовая функиия на $K \times K$ для любого компакта $K \subset A \backslash\left\{a_{1}, \ldots, a_{n}\right\}$. Eсли $w$ имеет особенности порядка $t<1$ в точках $\left(a_{i}, a_{i}\right)$, то результаты теоремы 2.1 остаются в силе.

Теперь при подходящих предположениях мы опишем асимптотику поведения взвешенных плотнейших упаковок в случае веса, зависящего от $N$. Пусть $\left\{v_{N}\right\}_{N=2}^{\infty}-$ последовательность неотрицательных весовых функций на $A \times A$. Скажем, что последовательность конфигураций $\left\{\omega_{N}\right\}_{N=2}^{\infty}$ на $A$, где $\# \omega_{N}=N$, $N=2,3, \ldots$, асимптотически оптимальна для этой последовательности весов, если

$$
\delta^{v_{N}}\left(\omega_{N}\right)=\delta_{N}^{v_{N}}(A)(1+o(1)), \quad N \rightarrow \infty .
$$

Теорема 2.4. Пусть $d \leqslant p$ иелье, и пусть $A \subset \mathbb{R}^{p}$ - произвольное бесконечное замкнутое $d$-спрямляемое множество. Пусть либо $w$ - CBD-вес на $A \times A$, либо множество $A$ и вес $w$ удовлетворяют условиям теоремь 2.3. Пусть $\left\{v_{N}\right\}_{N=2}^{\infty}-$ последовательность таких неотрицательных функиий на $A \times A$, чmo

$$
\left(1-\varepsilon_{N}\right) w(x, y) \leqslant v_{N}(x, y) \leqslant\left(1+\varepsilon_{N}\right) w(x, y), \quad(x, y) \in A \times A,
$$

где $\left\{\varepsilon_{N}\right\}_{N=2}^{\infty} \subset(0,1)$ - последовательность, сходящаяся $\kappa$ нулю. Тогда

$$
\lim _{N \rightarrow \infty} \delta_{N}^{v_{N}}(A) N^{1 / d}=C_{\infty, d}\left(\int_{A} w(x, x)^{d} d \mathscr{H}_{d}(x)\right)^{1 / d} .
$$


Более того, если $\mathscr{H}_{d}(A)>0$, то для всякой последовательности конфигураций $\omega_{N}:=\left\{x_{1}^{N}, \ldots, x_{N}^{N}\right\}, N=2,3, \ldots$, асимптотически оптимальной для последовательности весов $\left\{v_{N}\right\}_{N=2}^{\infty}$, выполняется

$$
\frac{1}{N} \sum_{k=1}^{N} \delta_{x_{k}^{N}} \stackrel{*}{\longrightarrow} h_{d}^{w}, \quad N \rightarrow \infty .
$$

\section{§ 3. Вспомогательные утверждения}

В дальнейшем нам будет удобно положить величину $\delta_{N}^{w}(A)$ равной нулю для конечных множеств при $N>\# A$. Для фиксированных натуральных чисел $d \leqslant p$ положим

$$
\underline{g}_{d}^{w}(A)=\liminf _{N \rightarrow \infty} \delta_{N}^{w}(A) N^{1 / d}, \quad \bar{g}_{d}^{w}(A)=\limsup _{N \rightarrow \infty} \delta_{N}^{w}(A) N^{1 / d}
$$

и

$$
g_{d}^{w}(A)=\lim _{N \rightarrow \infty} \delta_{N}^{w}(A) N^{1 / d}
$$

если этот предел существует. При $w \equiv 1$ обозначим вышеуказанные пределы соответственно через $g_{d}(A), \bar{g}_{d}(A)$ и $g_{d}(A)$. Для доказательства теорем 2.1 и 2.3 начнем со следующих аналогов лемм 1 и 2 из [18].

Лемма 3.1. Пусть $B$ и $D$ суть ограниченные подмножества $\mathbb{R}^{p}$, и пусть $w:(B \cup D) \times(B \cup D) \rightarrow[0, \infty]-$ произвольная весовая функция. Тогда

$$
\bar{g}_{d}^{w}(B \cup D)^{d} \leqslant \bar{g}_{d}^{w}(B)^{d}+\bar{g}_{d}^{w}(D)^{d} .
$$

Более того, если $\bar{g}_{d}^{w}(B), \bar{g}_{d}^{w}(D)<\infty$ и хотя бы одна из этих величин положительна, то

$$
\lim _{\mathscr{N} \ni N \rightarrow \infty} \frac{\#\left(\widetilde{\omega}_{N} \cap B\right)}{N}=\frac{\bar{g}_{d}^{w}(B)^{d}}{\bar{g}_{d}^{w}(B)^{d}+\bar{g}_{d}^{w}(D)^{d}}
$$

для всякой последовательности $\left\{\widetilde{\omega}_{N}\right\}_{N \in \mathscr{N}}$ таких $N$-точечных конфигураций в $B \cup D$, чmo

$$
\lim _{\mathscr{N} \ni N \rightarrow \infty} \delta^{w}\left(\widetilde{\omega}_{N}\right) N^{1 / d}=\left(\bar{g}_{d}^{w}(B)^{d}+\bar{g}_{d}^{w}(D)^{d}\right)^{1 / d},
$$

где $\mathscr{N}$ - бесконечное подмножество $\mathbb{N}$.

ДоказАТЕЛЬство. Если $B \cup D$ - конечное множество, то результат леммы тривиально выполнен. Пусть $\mathscr{N}_{1} \subset \mathbb{N}$ - бесконечное подмножество, и пусть $\left\{\omega_{N}\right\}_{N \in \mathscr{N}_{1}}$ - такая последовательность $N$-точечных конфигураций в $B \cup D$, что определен предел

$$
\alpha:=\lim _{\mathscr{N}_{1} \ni N \rightarrow \infty} \frac{\#\left(\omega_{N} \cap B\right)}{N} .
$$

Покажем, что

$$
\limsup _{\mathscr{N}_{1} \ni N \rightarrow \infty} \delta^{w}\left(\omega_{N}\right) N^{1 / d} \leqslant\left(\bar{g}_{d}^{w}(B)^{d}+\bar{g}_{d}^{w}(D)^{d}\right)^{1 / d}
$$


Пусть $N_{B}=\#\left(\omega_{N} \cap B\right)$ и $N_{D}=\#\left(\omega_{N} \backslash B\right), N \in \mathscr{N}_{1}$. Тогда

$$
\begin{aligned}
\delta^{w}\left(\omega_{N}\right) & =\min _{x \neq y \in \omega_{N}} w(x, y)|x-y| \\
& \leqslant \min \left\{\delta^{w}\left(\omega_{N} \cap B\right), \delta^{w}\left(\omega_{N} \backslash B\right)\right\} \leqslant \min \left\{\delta_{N_{B}}^{w}(B), \delta_{N_{D}}^{w}(D)\right\} .
\end{aligned}
$$

Если $0<\alpha<1$, то

$$
\begin{aligned}
& \limsup _{\mathscr{N}_{1} \ni N \rightarrow \infty} \delta^{w}\left(\omega_{N}\right) N^{1 / d} \\
& \quad \leqslant \min \left\{\limsup _{\mathscr{N}_{1} \ni N \rightarrow \infty} \delta_{N_{B}}^{w}(B) N_{B}^{1 / d}\left(\frac{N}{N_{B}}\right)^{1 / d}, \limsup _{\mathscr{N}_{1} \ni N \rightarrow \infty} \delta_{N_{D}}^{w}(D) N_{D}^{1 / d}\left(\frac{N}{N_{D}}\right)^{1 / d}\right\} \\
& \quad \leqslant \min \left\{\bar{g}_{d}^{w}(B) \alpha^{-1 / d}, \bar{g}_{d}^{w}(D)(1-\alpha)^{-1 / d}\right\} \leqslant\left(\bar{g}_{d}^{w}(B)^{d}+\bar{g}_{d}^{w}(D)^{d}\right)^{1 / d} .
\end{aligned}
$$

Если $\alpha=0$, то

$$
\begin{aligned}
& \limsup _{\mathscr{N}_{1} \ni N \rightarrow \infty} \delta^{w}\left(\omega_{N}\right) N^{1 / d} \\
& \quad \leqslant \limsup _{\mathscr{N}_{1} \ni N \rightarrow \infty} \delta^{w}\left(\omega_{N} \backslash B\right) N^{1 / d} \leqslant \limsup _{\mathscr{N}_{1} \ni N \rightarrow \infty} \delta_{N_{D}}^{w}(D) N_{D}^{1 / d}\left(\frac{N}{N_{D}}\right)^{1 / d} \\
& \leqslant \bar{g}_{d}^{w}(D) \leqslant\left(\bar{g}_{d}^{w}(B)^{d}+\bar{g}_{d}^{w}(D)^{d}\right)^{1 / d}
\end{aligned}
$$

Случай $\alpha=1$ разбирается аналогично, что и завершает обоснование (17).

Пусть теперь $\left\{\bar{\omega}_{N}\right\}_{N=2}^{\infty}$ - такая последовательность $N$-точечных конфигураций в $B \cup D$, что для достаточно большого $N$

$$
\begin{array}{ll}
\delta^{w}\left(\bar{\omega}_{N}\right)>\delta_{N}^{w}(B \cup D)-\frac{1}{N^{2 / d}} & \text { при } \delta_{N}^{w}(B \cup D)<\infty, \\
\delta^{w}\left(\bar{\omega}_{N}\right)>N & \text { при } \delta_{N}^{w}(B \cup D)=\infty, N \geqslant 2 .
\end{array}
$$

Поскольку $\left\{\#\left(\bar{\omega}_{N} \cap B\right) / N\right\}_{N=2}^{\infty}-$ ограниченная последовательность, то существует такое ограниченное подмножество $\mathscr{N}_{2} \subset \mathbb{N}$, что

$$
\bar{g}_{d}^{w}(B \cup D)=\lim _{\mathscr{N}_{2} \ni N \rightarrow \infty} \delta^{w}\left(\bar{\omega}_{N}\right) N^{1 / d},
$$

и существует предел

$$
\lim _{N_{2} \ni N \rightarrow \infty} \frac{\#\left(\bar{\omega}_{N} \cap B\right)}{N} .
$$

Тогда в силу (17)

$$
\bar{g}_{d}^{w}(B \cup D)=\lim _{\mathscr{N}_{2} \ni N \rightarrow \infty} \delta^{w}\left(\bar{\omega}_{N}\right) N^{1 / d} \leqslant\left(\bar{g}_{d}^{w}(B)^{d}+\bar{g}_{d}^{w}(D)^{d}\right)^{1 / d},
$$

что и доказывает (14).

Пусть теперь $\left\{\widetilde{\omega}_{N}\right\}_{N \in \mathscr{N}}$ - такая последовательность $N$-точечных конфигураций в $B \cup D$, что выполнено (16). Выберем подпоследовательность $\mathscr{N}_{3} \subset \mathscr{N}$, для которой существует предел

$$
\beta:=\lim _{N_{3} \ni N \rightarrow \infty} \frac{\#\left(\widetilde{\omega}_{N} \cap B\right)}{N} .
$$


Предположим, что $\bar{g}_{d}^{w}(B)$ и $\bar{g}_{d}^{w}(D)$ положительны. Используя проведенные рассуждения и (16), получаем

$$
\begin{aligned}
& \left(\bar{g}_{d}^{w}(B)^{d}+\bar{g}_{d}^{w}(D)^{d}\right)^{1 / d}=\lim _{\mathscr{N}_{3} \ni N \rightarrow \infty} \delta^{w}\left(\widetilde{\omega}_{N}\right) N^{1 / d} \\
& \quad \leqslant F(\beta):=\min \left\{\bar{g}_{d}^{w}(B) \beta^{-1 / d}, \bar{g}_{d}^{w}(D)(1-\beta)^{-1 / d}\right\} .
\end{aligned}
$$

Функция $F(\beta)$ достигает максимума на $[0,1]$ только в точке

$$
\alpha^{*}:=\frac{\bar{g}_{d}^{w}(B)^{d}}{\bar{g}_{d}^{w}(B)^{d}+\bar{g}_{d}^{w}(D)^{d}}
$$

и $F\left(\alpha^{*}\right)=\left(\bar{g}_{d}^{w}(B)^{d}+\bar{g}_{d}^{w}(D)^{d}\right)^{1 / d}$. Теперь в силу (18) с необходимостью получаем, что $\beta=\alpha^{*}$.

Допустим теперь, что $\bar{g}_{d}^{w}(B)=0$, но $\bar{g}_{d}^{w}(D)>0$. Если $\beta>0$, то

$$
\begin{aligned}
\bar{g}_{d}^{w}(D) & =\left(\bar{g}_{d}^{w}(B)^{d}+\bar{g}_{d}^{w}(D)^{d}\right)^{1 / d}=\lim _{\mathscr{N}_{3} \ni N \rightarrow \infty} \delta^{w}\left(\widetilde{\omega}_{N}\right) N^{1 / d} \\
& \leqslant \lim _{\mathscr{N}_{3} \ni N \rightarrow \infty} \delta^{w}\left(\widetilde{\omega}_{N} \cap B\right) N^{1 / d} \leqslant \bar{g}_{d}^{w}(B) \beta^{-1 / d}=0,
\end{aligned}
$$

что противоречит условию положительности $\bar{g}_{d}^{w}(D)$. Следовательно, $\beta=0=\alpha^{*}$. Если $\bar{g}_{d}^{w}(D)=0$, то $\beta=1=\alpha^{*}$, так что всегда $\beta=\alpha^{*}$. Поскольку подпоследовательность $\mathscr{N}_{3}$ произвольна, приходим к (15).

Лемма 3.2. Пусть $B$ и $D$ - подмножества $\mathbb{R}^{p}$, для которых $\operatorname{dist}(B, D)>0$. Пусть $w(x, y)>h$ при $(x, y) \in B \times D$ для некоторого $h>0$. Тогда

$$
\underline{g}_{d}^{w}(B \cup D)^{d} \geqslant \underline{g}_{d}^{w}(B)^{d}+\underline{g}_{d}^{w}(D)^{d} .
$$

ДокАзАтельство. Можно предположить, что $0<\underline{g}_{d}^{w}(B), \underline{g}_{d}^{w}(D)<\infty$, так как иначе лемма 3.2 тривиально выполняется. Пусть

$$
\alpha_{*}:=\frac{\underline{g}_{d}^{w}(B)^{d}}{\underline{g}_{d}^{w}(B)^{d}+\underline{g}_{d}^{w}(D)^{d}}
$$

и $a:=\operatorname{dist}(B, D) . \quad$ Пусть $\left\{\omega_{N}^{B}\right\}_{N=2}^{\infty}$ и $\left\{\omega_{N}^{D}\right\}_{N=2}^{\infty}-$ такие последовательности $N$-точечных конфигураций соответственно из $B$ и $D$, что

$$
\underline{g}_{d}^{w}(B)=\liminf _{N \rightarrow \infty} \delta^{w}\left(\omega_{N}^{B}\right) N^{1 / d}, \quad \underline{g}_{d}^{w}(D)=\liminf _{N \rightarrow \infty} \delta^{w}\left(\omega_{N}^{D}\right) N^{1 / d}
$$

(мы можем выбрать эти последовательности так же, как $\left\{\bar{\omega}_{N}\right\}$ в доказательстве леммы 3.1). Для всякого $N \in \mathbb{N}$ пусть $N_{B}:=\left\lfloor\alpha_{*} N\right\rfloor$ и $N_{D}:=N-\left\lfloor\alpha_{*} N\right\rfloor$, где $\lfloor t\rfloor$ - целая часть числа $t$. Тогда

$$
\delta_{N}^{w}(B \cup D) \geqslant \delta^{w}\left(\omega_{N_{B}}^{B} \cup \omega_{N_{D}}^{D}\right) \geqslant \min \left\{\delta^{w}\left(\omega_{N_{B}}^{B}\right), \delta^{w}\left(\omega_{N_{D}}^{D}\right), h a\right\} .
$$

Значит,

$$
\begin{aligned}
& \underline{g}_{d}^{w}(B \cup D)=\liminf _{N \rightarrow \infty} \delta_{N}^{w}(B \cup D) N^{1 / d} \\
& \quad \geqslant \min \left\{\liminf _{N \rightarrow \infty} \delta^{w}\left(\omega_{N_{B}}^{B}\right) N^{1 / d}, \liminf _{N \rightarrow \infty} \delta^{w}\left(\omega_{N_{D}}^{D}\right) N^{1 / d}, \lim _{N \rightarrow \infty} h a N^{1 / d}\right\}
\end{aligned}
$$




$$
\begin{aligned}
& =\min \left\{\liminf _{N \rightarrow \infty} \delta^{w}\left(\omega_{N_{B}}^{B}\right) N_{B}^{1 / d}\left(\frac{N}{N_{B}}\right)^{1 / d}, \liminf _{N \rightarrow \infty} \delta^{w}\left(\omega_{N_{D}}^{D}\right) N_{D}^{1 / d}\left(\frac{N}{N_{D}}\right)^{1 / d}\right\} \\
& \geqslant \min \left\{\underline{g}_{d}^{w}(B)\left(\alpha^{*}\right)^{-1 / d}, \underline{g}_{d}^{w}(D)\left(1-\alpha_{*}\right)^{-1 / d}\right\}=\left(\underline{g}_{d}^{w}(B)^{d}+\underline{g}_{d}^{w}(D)^{d}\right)^{1 / d},
\end{aligned}
$$

что завершает доказательство леммы 3.2 .

\section{§4. Случай веса, ограниченного на диагонали}

Теорема 2.1 - следствие следующего результата.

Лемма 4.1. Пусть $A \subset \mathbb{R}^{p}$ - компактное множество с $\mathscr{H}_{d}(A)<\infty, u$ пусть $w: A \times A \rightarrow[0, \infty]$ - CBD-весовая функция на $A \times A$. Предположим, далее, что для всякого компактного подмножества $K \subset A$ определен предел $g_{d}(K)$, заданный формулой

$$
g_{d}(K)=C_{\infty, d} \mathscr{H}_{d}(K)^{1 / d}
$$

Тогда:

(а) существует предел $g_{d}^{w}(A)$, заданный формулой

$$
g_{d}^{w}(A)=C_{\infty, d} \mathscr{H}_{d}^{w}(A)^{1 / d}
$$

(b) если последовательность $\left\{\widetilde{\omega}_{N}\right\}_{N=2}^{\infty}$, где $\widetilde{\omega}_{N}=\left\{x_{1}^{N}, \ldots, x_{N}^{N}\right\}$, реализует асимптотически плотнейшую $w$-упаковку на множестве $A$ u $\mathscr{H}_{d}(A)>0$, mo

$$
\frac{1}{N} \sum_{k=1}^{N} \delta_{x_{k}^{N}} \stackrel{*}{\longrightarrow} h_{d}^{w}, \quad N \rightarrow \infty .
$$

ДокАЗАТЕЛьство. Для доказательства первой части леммы (соотношения (20)) разобъем $A$ на непересекающиеся “куски" малого диаметра и оценим радиус $w$-наилучшей упаковки $A$, заменяя $w$ ее супремумом или инфимумом на каждом из кусков и применяя леммы 3.1 и 3.2 .

Предположим для $\delta>0$, что $\mathscr{P}_{\delta}$ - такое разбиение $A$, что $\operatorname{diam} P \leqslant \delta$ и $\mathscr{H}_{d}(\bar{P})=\mathscr{H}_{d}(P)$ при $P \in \mathscr{P}_{\delta}$, где $\bar{B}$ есть замыкание множества $B$. Для всякого $P \in \mathscr{P}_{\delta}$ выберем такое замкнутое $Q_{P} \subset P$, чтобы $\mathscr{Q}_{\delta}:=\left\{Q_{P}: P \in \mathscr{P}_{\delta}\right\}$ удовлетворяло соотношению

$$
\sum_{P \in \mathscr{P}_{\delta}} \mathscr{H}_{d}\left(Q_{P}\right) \geqslant \mathscr{H}_{d}(A)-\delta .
$$

Мы можем построить пример таких наборов $\mathscr{P}_{\delta}$ и $\mathscr{Q}_{\delta}$ следующим образом. Пусть $G_{j}[t]$ - гиперплоскость в $\mathbb{R}^{p}$, образованная точками с $j$-й координатой, равной $t$. Если $(-a, a)^{p}-$ куб, содержащий $A$, то для $\mathbf{i}=\left(i_{1}, \ldots, i_{p}\right) \in$ $\{1, \ldots, m\}^{p}$ положим

$$
R_{\mathbf{i}}:=\left[t_{i_{1}-1}^{1}, t_{i_{1}}^{1}\right) \times \cdots \times\left[t_{i_{p}-1}^{p}, t_{i_{p}}^{p}\right),
$$


где число $m$ и разбиения $-a=t_{0}^{j}<t_{1}^{j}<\cdots<t_{m}^{j}=a, j=1, \ldots, p$, выбраны так, чтобы диаметр каждого $R_{\mathbf{i}}, \mathbf{i} \in\{1, \ldots, m\}^{p}$, оказался меньше $\delta$ и $\mathscr{H}_{d}\left(G_{j}\left[t_{i}^{j}\right] \cap A\right)=0$ для всех $i$ и $j$. (Поскольку $\mathscr{H}_{d}(A)<\infty$, то множество $t$, для которых $\mathscr{H}_{d}\left(G_{j}[t] \cap A\right)>0$, не более чем счетно.) Теперь можно выбрать

$$
\mathscr{P}_{\delta}=\left\{R_{\mathbf{i}} \cap A: \mathbf{i} \in\{1, \ldots, m\}^{p}\right\}
$$

и $\gamma \in(0,1)$, достаточно близкое к 1 , такими, чтобы $(22)$ выполнялось при $\mathscr{Q}_{\delta}=\left\{Q_{\mathbf{i}}: \mathbf{i} \in\{1, \ldots, m\}^{p}\right\}$, где $Q_{\mathbf{i}}=\left(\gamma\left(\bar{R}_{\mathbf{i}}-c_{\mathbf{i}}\right)+c_{\mathbf{i}}\right) \cap A$ и $c_{\mathbf{i}}-$ центр $R_{\mathbf{i}}$.

Для $B \subset A$ положим

$$
\bar{w}_{B}:=\sup _{x, y \in B} w(x, y), \quad \underline{w}_{B}:=\inf _{x, y \in B} w(x, y)
$$

и введем простые функции

$$
\bar{w}_{\delta}(x):=\sum_{P \in \mathscr{P}_{\delta}} \bar{w}_{P} \chi_{P}(x), \quad \underline{w}_{\delta}(x):=\sum_{P \in \mathscr{P}_{\delta}} \underline{w}_{P} \chi_{P}(x),
$$

где $\chi_{K}$ - характеристическая функция множества $K$. Поскольку расстояние между любой парой множеств из $\mathscr{Q}_{\delta}$ строго положительно, то, применяя лемму 3.2 и равенство (19), получим

$$
\begin{aligned}
\underline{g}_{d}^{w}(A)^{d} & \geqslant \underline{g}_{d}^{w}\left(\bigcup_{Q \in \mathscr{Q}_{\delta}} Q\right)^{d} \geqslant \sum_{Q \in \mathscr{Q}_{\delta}} \underline{w}_{Q}^{d} \underline{g}_{d}(Q)^{d} \\
& \geqslant C_{\infty, d}^{d} \sum_{Q \in \mathscr{Q}_{\delta}} \underline{w}_{Q}^{d} \mathscr{H}_{d}(Q) \geqslant C_{\infty, d}^{d} \int_{\bigcup_{Q \in \mathcal{Q}_{\delta}} Q} \underline{w}_{\delta}(x)^{d} d \mathscr{H}_{d}(x) .
\end{aligned}
$$

Мы полагаем здесь $\delta_{N}^{w}(Q)=0$ при $\# Q$, равном 0 или 1 .

С другой стороны, по лемме 3.1 , теореме 1.1 и в силу свойств разбиения $\mathscr{P}_{\delta}$ мы получаем следующий результат:

$$
\begin{aligned}
\bar{g}_{d}^{w}(A)^{d} & =\bar{g}_{d}^{w}\left(\bigcup_{P \in \mathscr{P}_{\delta}} \bar{P}\right)^{d} \leqslant \sum_{P \in \mathscr{P}_{\delta}} \bar{g}_{d}^{w}(\bar{P})^{d} \leqslant \sum_{P \in \mathscr{P}_{\delta}} \bar{w}_{P}^{d} \bar{g}_{d}(\bar{P})^{d} \\
& =C_{\infty, d}^{d} \sum_{P \in \mathscr{P}_{\delta}} \bar{w}_{P}^{d} \mathscr{H}_{d}(\bar{P})=C_{\infty, d}^{d} \sum_{P \in \mathscr{P}_{\delta}} \bar{w}_{P}^{d} \mathscr{H}_{d}(P) \\
& =C_{\infty, d}^{d} \int_{A} \bar{w}_{\delta}(x)^{d} d \mathscr{H}_{d}(x) .
\end{aligned}
$$

Поскольку $w$ - CBD-вес на $A \times A$, то существует окрестность $G$ диагонали $D(A)$, в которой $\tau:=\sup _{G} w<\infty$. При достаточно малом $\delta>0$ получаем $P \times P \subset G$ для всякого $P \in \mathscr{P}_{\delta}$, так что $\underline{w}_{\delta}(x) \leqslant w(x, x) \leqslant \bar{w}_{\delta}(x) \leqslant \tau$ при $x \in A$. Более того, $w$ непрерывна в $(x, x) \in D(A)$ для $\mathscr{H}_{d}$-почти всех $x \in A$, так что $\bar{w}_{\delta}(x)$ и $\underline{w}_{\delta}(x)$ сходятся к $w(x, x)$ при $\delta \rightarrow 0$ для любого такого $x$. Значит, оба интеграла

$$
\int_{\bigcup_{Q \in \mathcal{Q}_{\delta}} Q} \underline{w}_{\delta}(x)^{d} d \mathscr{H}_{d}(x), \quad \int_{A} \bar{w}_{\delta}(x)^{d} d \mathscr{H}_{d}(x)
$$


сходятся к $\mathscr{H}_{d}^{w}(A)$ при $\delta \rightarrow 0$ по теореме Лебега о сходимости, так что, используя неравенство (23) и (24), получаем (20).

Докажем теперь (21). Пусть $\mathscr{H}_{d}(A)>0$, и пусть $\widetilde{\omega}_{N}:=\left\{x_{1}^{N}, \ldots, x_{N}^{N}\right\}$, $N \in \mathbb{N},-$ последовательность конфигураций из $A$, реализующая асимптотически $w$-плотнейшую упаковку. Выберем подмножество $B \subset A$, относительная граница которого в $A$ (мы обозначим ее $\left.\partial_{A} B\right)$ имеет $\mathscr{H}_{d}$-меру нуль. Покажем, что

$$
\lim _{N \rightarrow \infty} \frac{\#\left(\widetilde{\omega}_{N} \cap B\right)}{N}=h_{d}^{w}(B) .
$$

Заметим, что $\bar{B}$ и $\overline{A \backslash B}$ суть компакты конечной $\mathscr{H}_{d}$-меры и что ограничение $w$ на каждый из них остается CBD-весом. Всякое компактное подмножество этих множеств - компактное подмножество $A$, так что эти множества удовлетворяют условиям леммы 4.1. Более того, $\mathscr{H}_{d}^{w}\left(\partial_{A} \bar{B}\right)=\mathscr{H}_{d}^{w}\left(\partial_{A} \overline{A \backslash B}\right)=0$. Таким образом,

$$
g_{d}^{w}(\bar{B})=C_{\infty, d} \mathscr{H}_{d}^{w}(\bar{B})^{1 / d}, \quad g_{d}^{w}(\overline{A \backslash B})=C_{\infty, d} \mathscr{H}_{d}^{w}(\overline{A \backslash B})^{1 / d},
$$

и, учитывая соотношение (20), получаем

$$
\begin{aligned}
\lim _{N \rightarrow \infty} & \delta^{w}\left(\widetilde{\omega}_{N}\right) N^{1 / d}=\lim _{N \rightarrow \infty} \delta_{N}^{w}(A) N^{1 / d}=g_{d}^{w}(A)=C_{\infty, d} \mathscr{H}_{d}^{w}(A)^{1 / d} \\
& =C_{\infty, d}\left(\mathscr{H}_{d}^{w}(\bar{B})+\mathscr{H}_{d}^{w}(\overline{A \backslash B})\right)^{1 / d}=\left(g_{d}^{w}(\bar{B})^{d}+g_{d}^{w}(\overline{A \backslash B})^{d}\right)^{1 / d} .
\end{aligned}
$$

В силу соотношения (16) из леммы 3.1

$$
\lim _{N \rightarrow \infty} \frac{\#\left(\widetilde{\omega}_{N} \cap \bar{B}\right)}{N}=\frac{g_{d}^{w}(\bar{B})^{d}}{g_{d}^{w}(\bar{B})^{d}+g_{d}^{w}(\overline{A \backslash B})^{d}}=h_{d}^{w}(\bar{B})=h_{d}^{w}(B) .
$$

Поскольку (26) выполнено для всякого замкнутого подмножества $A$ с границей (относительно $A$ ) $\mathscr{H}_{d}$-меры нуль, то

$$
\lim _{N \rightarrow \infty} \frac{\#\left(\widetilde{\omega}_{N} \cap(\bar{B} \backslash B)\right)}{N} \leqslant \lim _{N \rightarrow \infty} \frac{\#\left(\widetilde{\omega}_{N} \cap \partial_{A} B\right)}{N}=h_{d}^{w}\left(\partial_{A} B\right)=0 .
$$

Следовательно, равенство (25) выполняется для всех $B \subset A$ с границей (относительно $A$ ) нулевой $\mathscr{H}_{d}$-меры. Как указано в $\S 1$, отсюда следует $(21)$, что и завершает доказательство.

ДОКАЗАТЕЛЬСТВо ТЕОРЕМЫ 2.1. Поскольку $A$ - спрямляемый компакт, то $\mathscr{H}_{d}(A)<\infty$, и всякое его замкнутое подмножество $K$ также является $d$-спрямляемым компактом. По теореме 1.1 равенство (19) выполнено для $K$. Теорема 2.1 следует теперь из леммы 4.1.

\section{§ 5. Случай веса с особенностями на диагонали}

В этом параграфе мы исследуем асимптотическое поведение $\delta_{N}^{w}(A)$. Из условия теоремы 2.3 вытекает, что величина

$$
\mathscr{H}_{d}^{w}(A)=\int_{A} w(x, x)^{d} d \mathscr{H}_{d}(x)
$$


конечна и положительна (см. доказательство ниже), так что $g_{d}^{w}(A)$ имеет те же свойства. Следующая лемма, в которой предполагается регулярность снизу, оказывается важным компонентом доказательства теоремы 2.3. В духе определения $\alpha$-регулярности (10) скажем, что множество $K \subset \mathbb{R}^{p} \alpha$-регулярно снизу в $a \in K$, если существуют такие положительные постоянные $C_{0}$ и $r_{0}$, что

$$
C_{0}^{-1} r^{\alpha} \leqslant \mathscr{H}_{\alpha}(K \cap B(x, r)), \quad x \in K \cap B\left(a, r_{0}\right), \quad 0<r<r_{0} .
$$

Лемма 5.1. Пусть $\alpha>0$, и пусть $K-$ компакт в $\mathbb{R}^{p}, \alpha$-регулярный снизу в $а \in K$. Пусть $w: K \times K \rightarrow[0, \infty]$ - вес с особенностью порядка не более $t<1$ в а. Тогда существует такая константа $C_{1}=C_{1}(w, K, t, \alpha)$, что для достаточно мальх $\lambda$

$$
\bar{g}_{\alpha}^{w}(K \cap B(a, \lambda)) \leqslant C_{1}\left(\int_{K \cap B(a, 2 \lambda)} \frac{1}{|x-a|^{t \alpha}} d \mathscr{H}_{\alpha}(x)\right)^{1 / \alpha} .
$$

ДокАЗАТЕЛЬСтво. Возьмем $r_{0}$ и $C_{0}$ из определения $\alpha$-регулярности снизу множества $K$ в точке $a$, и пусть $C$ и $\delta$ такие же, как в определении особенности $w$ порядка не более $t<1$ в точке $a$.

Выберем $0<\lambda<\min \left\{r_{0}, \delta\right\}$. Пусть $\omega_{N}=\left\{x_{1}, \ldots, x_{N}\right\}-$ произвольная конфигурация $N$ различных точек из $K \cap B(a, \lambda)$. Для $i=1, \ldots, N$ пусть $\rho_{i}=\left|x_{i}-a\right|, r_{i}=\min _{j: j \neq i}\left|x_{j}-x_{i}\right|$, и пусть $y_{i}-$ такая точка в $\omega_{N}$, что $\left|x_{i}-y_{i}\right|=r_{i}$.

Поскольку $K \cap B(a, \lambda)$ ограничено, то существует не более $L-1$ таких точек $x_{i} \in \omega_{N}$ (например, можно положить $L=3^{p}+1$ ), что $r_{i} \geqslant \lambda$. Перенумеруем точки из $\omega_{N}$ так, чтобы $\rho_{N} \leqslant \rho_{i}, i=1, \ldots, N-1$, и $r_{i}<\lambda, i=1, \ldots, N-L$. Тогда

$$
\delta^{w}\left(\omega_{N}\right)=\min _{i \neq j} w\left(x_{i}, x_{j}\right)\left|x_{i}-x_{j}\right| \leqslant \min _{i=1, \ldots, N-L} w\left(x_{i}, y_{i}\right)\left|x_{i}-y_{i}\right|
$$

Поскольку $x_{i}, y_{i} \in K \cap B(a, \lambda) \subset K \cap B(a, \delta)$ для всякого $i=1, \ldots, N-L$, то имеем

$$
w\left(x_{i}, y_{i}\right) \leqslant \frac{C}{\left|x_{i}-a\right|^{t}} .
$$

Следовательно,

$$
\delta^{w}\left(\omega_{N}\right) \leqslant \min _{i=1, \ldots, N-L} \frac{C r_{i}}{\left|x_{i}-a\right|^{t}}=\min _{i=1, \ldots, N-L} \frac{C r_{i}}{\rho_{i}^{t}} \leqslant C\left(\frac{1}{N-L} \sum_{i=1}^{N-L} \frac{r_{i}^{\alpha}}{\rho_{i}^{t \alpha}}\right)^{1 / \alpha} .
$$

При $i=1, \ldots, N-1$

$$
r_{i}=\min _{j: j \neq i}\left|x_{j}-x_{i}\right| \leqslant\left|x_{i}-a\right|+\min _{j: j \neq i}\left|a-x_{j}\right| \leqslant \rho_{i}+\rho_{N} \leqslant 2 \rho_{i} .
$$

Для $x \in B\left(x_{i}, r_{i} / 2\right), i=1, \ldots, N-1$, мы также имеем

$$
|x-a| \leqslant\left|x-x_{i}\right|+\left|x_{i}-a\right| \leqslant \frac{r_{i}}{2}+\rho_{i} \leqslant 2 \rho_{i} .
$$


Учитывая $\alpha$-регулярность снизу множества $K$ в точке $a$, нетрудно видеть, что

$$
\begin{aligned}
\frac{r_{i}^{\alpha}}{\rho_{i}^{t \alpha}} & \leqslant 2^{\alpha} C_{0} \mathscr{H}_{\alpha}\left(K \cap B\left(x_{i}, \frac{r_{i}}{2}\right)\right) \frac{1}{\rho_{i}^{t \alpha}} \\
& \leqslant 2^{\alpha} C_{0} \int_{K \cap B\left(x_{i}, r_{i} / 2\right)} \frac{1}{\rho_{i}^{t \alpha}} d \mathscr{H}_{\alpha}(x) \leqslant 2^{\alpha(t+1)} C_{0} \int_{K \cap B\left(x_{i}, r_{i} / 2\right)} \frac{1}{|x-a|^{t \alpha}} d \mathscr{H}_{\alpha}(x) .
\end{aligned}
$$

Таким образом,

$$
\delta^{w}\left(\omega_{N}\right) \leqslant 2^{t+1} C_{0}^{1 / \alpha} C(N-L)^{-1 / \alpha}\left(\sum_{i=1}^{N-L} \int_{K \cap B\left(x_{i}, r_{i} / 2\right)} \frac{1}{|x-a|^{t \alpha}} d \mathscr{H}_{\alpha}(x)\right)^{1 / \alpha} .
$$

Поскольку $\omega_{N}$ - произвольный набор $N$ точек из $K \cap B(a, \lambda)$ и $B\left(x_{i}, r_{i} / 2\right) \cap$ $B\left(x_{j}, r_{j} / 2\right)=\varnothing, i \neq j$, мы можем записать

$$
\delta_{N}^{w}(K \cap B(a, \lambda)) \leqslant 2^{t+1} C_{0}^{1 / \alpha} C(N-L)^{-1 / \alpha}\left(\int_{K \cap B(a, 2 \lambda)} \frac{1}{|x-a|^{t \alpha}} d \mathscr{H}_{\alpha}(x)\right)^{1 / \alpha} .
$$

Следовательно,

$$
\begin{aligned}
& \bar{g}_{\alpha}^{w}(K\cap B(a, \lambda))=\limsup _{N \rightarrow \infty} \delta_{N}^{w}(K \cap B(a, \lambda))(N-L)^{1 / \alpha} \\
& \leqslant C_{1}\left(\int_{K \cap B(a, 2 \lambda)} \frac{1}{|x-a|^{t \alpha}} d \mathscr{H}_{\alpha}(x)\right)^{1 / \alpha}
\end{aligned}
$$

где $C_{1}=2^{t+1} C_{0}^{1 / \alpha} C$, что и завершает доказательство леммы 5.1.

ДоказАТЕЛЬСтво теоремы 2.3. Если $K \alpha$-регулярно, $0<\alpha \leqslant d$, а не просто $\alpha$-регулярно снизу в $a$, то интеграл

$$
\int_{K} \frac{1}{|x-a|^{t \alpha}} d \mathscr{H}_{\alpha}(x)
$$

конечен (ср. [15; с. 109]), и в силу абсолютной непрерывности интеграла Лебега получаем

$$
\lim _{\lambda \rightarrow 0} \int_{K \cap B(a, 2 \lambda)} \frac{1}{|x-a|^{t \alpha}} d \mathscr{H}_{\alpha}(x)=0 .
$$

Теперь по лемме 5.1 получаем, что $\lim _{\lambda \rightarrow 0} \bar{g}_{\alpha}^{w}(K \cap B(a, \lambda))=0$ для всякого $0<\alpha \leqslant d$. Следовательно, $\lim _{\lambda \rightarrow 0} \bar{g}_{d}^{w}(K \cap B(a, \lambda))=0$.

Из $\alpha_{i}$-регулярности $A$ в $a_{i}$ и того факта, что $w$ имеет в $a_{i}$ особенность порядка не выше $t<1, i=1, \ldots, n$, вытекает, что

$$
\int_{A} w(x, x)^{d} d \mathscr{H}_{d}(x)<\infty .
$$

Пусть $\varepsilon>0$. По леммам 5.1 и 3.1 можно найти такое $\delta>0$, что для $B_{\delta}:=$ $\bigcup_{i=1}^{n} A \cap B\left(a_{i}, \delta\right)$ мы имеем $\bar{g}_{d}^{w}\left(B_{\delta}\right)<\varepsilon$, и при $A_{\delta}:=A \backslash B_{\delta}$

$$
\mathscr{H}_{d}^{w}\left(A_{\delta}\right)=\int_{A_{\delta}} w(x, x)^{d} d \mathscr{H}_{d}(x) \geqslant(1-\varepsilon) \mathscr{H}_{d}^{w}(A) .
$$


Поскольку $w$ - CBD-вес на $A_{\delta} \times A_{\delta}$, то по теореме 2.1 получаем, что $g_{d}^{w}\left(A_{\delta}\right)$ определено и дано равенством $g_{d}^{w}\left(A_{\delta}\right)=C_{\infty, d} \mathscr{H}_{d}^{w}\left(A_{\delta}\right)^{1 / d}$. Теперь по лемме 3.1

$$
\begin{aligned}
\bar{g}_{d}^{w}(A) & \leqslant\left(\bar{g}_{d}^{w}\left(A_{\delta}\right)^{d}+\bar{g}_{d}^{w}\left(B_{\delta}\right)^{d}\right)^{1 / d} \\
& \leqslant\left(C_{\infty, d}^{d} \mathscr{H}_{d}^{w}\left(A_{\delta}\right)+\varepsilon^{d}\right)^{1 / d} \leqslant\left(C_{\infty, d}^{d} \mathscr{H}_{d}^{w}(A)+\varepsilon^{d}\right)^{1 / d} .
\end{aligned}
$$

Кроме того,

$$
\underline{g}_{d}^{w}(A) \geqslant \underline{g}_{d}^{w}\left(A_{\delta}\right)=C_{\infty, d} \mathscr{H}_{d}^{w}\left(A_{\delta}\right)^{1 / d} \geqslant C_{\infty, d}(1-\varepsilon)^{1 / d} \mathscr{H}_{d}^{w}(A)^{1 / d} .
$$

Взяв $\varepsilon \rightarrow 0$ в неравенствах $(28)$ и $(29)$, мы видим, что $g_{d}^{w}(A)$ определено и равенство (8) также выполняется в условиях теоремы 2.3.

Покажем, что (9) выполняется для веса с сингулярностями. Пусть $B \subset A$ - такое множество, что $\mathscr{H}_{d}\left(\partial_{A} B\right)=0$. Для всякого $\delta>0$, для которого $\mathscr{H}_{d}\left(A \cap \partial_{\mathbb{R}^{p}} B\left(a_{i}, \delta\right)\right)=0, i=1, \ldots, n$ (это условие может нарушаться для не более чем счетного числа $\delta)$, рассмотрим множества $B_{1}=B \cup\left(\bigcup_{i=1}^{n} A \cap B\left(a_{i}, \delta\right)\right)$ и $A \backslash B_{1}$. Заметим, что $\mathscr{H}_{d}^{w}\left(\partial_{A} B_{1}\right)=\mathscr{H}_{d}^{w}\left(\partial_{A} A \backslash B_{1}\right)=0$. Оба множества $d$-спрямляемые. Поскольку $\overline{A \backslash B_{1}}$ тоже является компактным подмножеством $A \backslash\left\{a_{1}, \ldots, a_{n}\right\}$, то $w$ - CBD-весовая функция на $\overline{A \backslash B_{1}} \times \overline{A \backslash B_{1}}$. Теперь по теореме 2.1 получаем

$$
g_{d}^{w}\left(\overline{A \backslash B_{1}}\right)=C_{\infty, d} \mathscr{H}_{d}^{w}\left(A \backslash B_{1}\right)^{1 / d} .
$$

Множество $\bar{B}_{1}$ компактно, и при достаточно малом $\delta>0$ оно $\alpha_{i}$-регулярно в $a_{i}$, $i=1, \ldots, n$. Ограничение $w$ на $\bar{B}_{1} \times \bar{B}_{1}$ оказывается весом с особенностями порядка не выше $t<1$ в $a_{1}, \ldots, a_{n}$, так что по (8) получаем

$$
g_{d}^{w}\left(\bar{B}_{1}\right)=C_{\infty, d} \mathscr{H}_{d}^{w}\left(B_{1}\right)^{1 / d} .
$$

Пусть $\left\{\bar{\omega}_{N}\right\}_{N=2}^{\infty}, \# \bar{\omega}_{N}=N,-$ последовательность, реализующая $w$-плотнейшую упаковку. Тогда

$$
\begin{aligned}
\lim _{N \rightarrow \infty} & \delta^{w}\left(\bar{\omega}_{N}\right) N^{1 / d}=g_{d}^{w}(A)=C_{\infty, d} \mathscr{H}_{d}^{w}(A)^{1 / d} \\
\quad & =C_{\infty, d}\left(\mathscr{H}_{d}^{w}\left(B_{1}\right)+\mathscr{H}_{d}^{w}\left(A \backslash B_{1}\right)\right)^{1 / d}=\left(g_{d}^{w}\left(\bar{B}_{1}\right)^{d}+g_{d}^{w}\left(\overline{A \backslash B_{1}}\right)^{d}\right)^{1 / d} .
\end{aligned}
$$

Поскольку $\mathscr{H}_{d}^{w}(A)>0$, то одна из величин $\mathscr{H}_{d}^{w}\left(\bar{B}_{1}\right)$ и $\mathscr{H}_{d}^{w}\left(\overline{A \backslash B_{1}}\right)$ должна быть положительной. Используя соотношение (15) из леммы 3.1 , получаем

$$
\limsup _{N \rightarrow \infty} \frac{\#\left(\bar{\omega}_{N} \cap B\right)}{N} \leqslant \lim _{N \rightarrow \infty} \frac{\#\left(\bar{\omega}_{N} \cap \bar{B}_{1}\right)}{N}=\frac{g_{d}^{w}\left(\bar{B}_{1}\right)^{d}}{g_{d}^{w}\left(\bar{B}_{1}\right)^{d}+g_{d}^{w}\left(\overline{A \backslash B_{1}}\right)^{d}}=h_{d}^{w}\left(B_{1}\right) .
$$

Так как $\lim _{\delta \rightarrow 0} h_{d}^{w}\left(B_{1}\right)=h_{d}^{w}(B)$, то

$$
\limsup _{N \rightarrow \infty} \frac{\#\left(\bar{\omega}_{N} \cap B\right)}{N} \leqslant h_{d}^{w}(B) .
$$

Повторяя эти рассуждения для $B_{1}=(A \backslash B) \cup\left(\bigcup_{i=1}^{n} A \cap B\left(a_{i}, \delta\right)\right)$, мы видим, что

$$
\limsup _{N \rightarrow \infty} \frac{\#\left(\bar{\omega}_{N} \cap(A \backslash B)\right)}{N} \leqslant h_{d}^{w}(A \backslash B) .
$$


Следовательно,

$$
\liminf _{N \rightarrow \infty} \frac{\#\left(\bar{\omega}_{N} \cap B\right)}{N} \geqslant h_{d}^{w}(B) .
$$

Вместе с (30) это соотношение показывает, что

$$
\lim _{N \rightarrow \infty} \frac{\#\left(\bar{\omega}_{N} \cap B\right)}{N}=h_{d}^{w}(B)
$$

откуда следует, что (9) также выполнено в предположениях теоремы 2.3.

ДОКАЗАТЕЛЬСТВо ТЕОРЕМЫ 2.4. В силу неравенств (11) для всякой конфигурации $\omega_{N}:=\left\{x_{1}, \ldots, x_{N}\right\} \subset A$ получаем

$$
\begin{aligned}
\delta^{v_{N}}\left(\omega_{N}\right) & =\min _{i \neq j} v_{N}\left(x_{i}, x_{j}\right)\left|x_{i}-x_{j}\right| \\
& \geqslant\left(1-\varepsilon_{N}\right) \min _{i \neq j} w\left(x_{i}, x_{j}\right)\left|x_{i}-x_{j}\right|=\left(1-\varepsilon_{N}\right) \delta^{w}\left(\omega_{N}\right) .
\end{aligned}
$$

Аналогично,

$$
\delta^{v_{N}}\left(\omega_{N}\right) \leqslant\left(1+\varepsilon_{N}\right) \delta^{w}\left(\omega_{N}\right)
$$

Таким образом,

$$
\left(1-\varepsilon_{N}\right) \delta_{N}^{w}(A) \leqslant \delta_{N}^{v_{N}}(A) \leqslant\left(1+\varepsilon_{N}\right) \delta_{N}^{w}(A),
$$

и по теореме 2.1 или 2.3 получаем

$$
\lim _{N \rightarrow \infty} \delta_{N}^{v_{N}}(A) N^{1 / d}=\lim _{N \rightarrow \infty} \delta_{N}^{w}(A) N^{1 / d}=C_{\infty, d}\left(\mathscr{H}_{d}^{w}(A)\right)^{1 / d} .
$$

Пусть теперь $\left\{\omega_{N}\right\}_{N=2}^{\infty}-$ асимптотически оптимальная последовательность конфигураций для последовательности весов $\left\{v_{N}\right\}_{N=2}^{\infty}$. Учитывая (33), получаем

$$
\delta^{v_{N}}\left(\omega_{N}\right)=\delta_{N}^{v_{N}}(A)(1+o(1))=\delta_{N}^{w}(A)(1+o(1)), \quad N \rightarrow \infty .
$$

Из соотношений (31) и (32) следует также, что

$$
\delta^{v_{N}}\left(\omega_{N}\right)=\delta^{w}\left(\omega_{N}\right)(1+o(1)), \quad N \rightarrow \infty .
$$

Тогда

$$
\delta^{w}\left(\omega_{N}\right)=\delta_{N}^{w}(A)(1+o(1)), \quad N \rightarrow \infty,
$$

так что последовательность $\left\{\omega_{N}\right\}_{N=2}^{\infty}$ реализует асимптотически $w$-плотнейшую упаковку на $A$. Снова применяя теорему 2.1 или 2.3 , мы приходим к (12).

\section{Список литературы}

[1] L. Fejes Tóth, Lagerungen in der Ebene, auf der Kugel und im Raum, Springer-Verlag, Berlin-Göttingen-Heidelberg, 1953; рус. пер.: Л. Тот Фейеш, Расположения на плоскости, на сфере и в пространстве, Физматгиз, М., 1958.

[2] L. Fejes Tóth, Regular figures, Macmillan, New York; Pergamon Press, Oxford, 1964. 
[3] C. A. Rogers, Packing and covering, Cambridge Tracts in Mathematics and Mathematical Physics, 54, Cambridge Univ. Press, New York, 1964; рус. пер.: К. Роджерс, Укладки и покрытия, Мир, М., 1968.

[4] J. H. Conway, N. J. A. Sloane, Sphere packings, lattices and groups, Grundlehren Math. Wiss., 290, Springer-Verlag, New York, 1999; pyc. пер.: Дж. Конвей, Н. Слоэн, Упаковки шаров, решетки и группы, Мир, М., 1990.

[5] K. Böröczky, jr., Finite packing and covering, Cambridge Tracts in Math., 154, Cambridge Univ. Press, Cambridge, 2004.

[6] Th. C. Hales, "A proof of the Kepler conjecture", Ann. of Math. (2), 162:3 (2005), $1065-1185$.

[7] V.F. Babenko, "On the optimal error bound for cubature formulae on certain classes of continuous functions", Anal. Math., 3:1 (1977), 3-9.

[8] А. Н. Колмогоров, В. М. Тихомиров, " $\varepsilon$-энтропия и $\varepsilon$-емкость множеств в функциональных пространствах", УМH, 14:2 (1959), 3-86; англ. пер.: А. N. Kolmogorov, V.M. Tikhomirov, " $\varepsilon$-entropy and $\varepsilon$-capacity of sets in functional spaces", Amer. Math. Soc. Transl. (2), 17 (1961), 227-364.

[9] V. Afraimovich, L. Glebsky, "Measures of $\varepsilon$-complexity", Taiwanese J. Math., 9:3 (2005), 397-409.

[10] E. V. Chornaya, "On the optimization of weighted cubature formulae on certain classes of continuous functions", East J. Approx., 1:1 (1995), 47-60.

[11] W. Habicht, B. L. van der Waerden, "Lagerung von Punkten auf der Kugel", Math. Ann., 123:1 (1951), 223-234.

[12] B. L. van der Waerden, "Punkte auf der Kugel. Drei Zusätze", Math. Ann., 125:1 (1952), 213-222.

[13] S. V. Borodachov, D. P. Hardin, E. B. Saff, "Asymptotics of best-packing on rectifiable sets", Proc. Amer. Math. Soc., 135:8 (2007), 2369-2380.

[14] H. Federer, Geometric measure theory, Die Grundlehren der mathematischen Wissenschaften, 153, Springer-Verlag, Berlin-Heidelberg-New York, 1969; рус. пер.: Г. Федерер, Геометрическая теория мерь, Наука, М., 1987.

[15] P. Mattila, Geometry of sets and measures in Euclidean spaces. Fractals and rectifiability, Cambridge Stud. Adv. Math., 44, Cambridge Univ. Press, Cambridge, 1995.

[16] K. Böröczky, "Packing of spheres in spaces of constant curvature", Acta Math. Acad. Sci. Hungar., 32:3-4 (1978), 243-261.

[17] L. Bowen, Ch. Radin, "Densest packing of equal spheres in hyperbolic space", Discrete Comput. Geom., 29:1 (2003), 23-39.

[18] S. V. Borodachov, D. P. Hardin, E. B. Saff, "Asymptotics for discrete weighted minimal Riesz energy problems on rectifiable sets", Trans. Amer. Math. Soc., 360:3 (2008), 1559-1580.

[19] В.А.Юдин, "Минимум потенциальной энергии точечной системы зарядов", Дискрет. матем., 4:2 (1992), 115-121; англ. пер. V.A. Yudin, "The minimum of potential energy of a system of point charges", Discrete Math. Appl., 3:1 (1993), 75-81.

[20] Н.Н. Андреев, "Расположение точек на сфере с минимальной энергией”, Теория приближений. Гармонический анализ, Тр. МИАН, 219, Наука, М., 1997, 27-31; англ. пер. N. N. Andreev, "Disposition points on a sphere with minimum of energy", Proc. Steklov Inst. Math., 219 (1997), 20-24.

[21] A. V. Kolushov, V.A. Yudin, "Extremal dispositions of points on the sphere", Anal. Math., 23:1 (1997), 25-34.

[22] D. P. Hardin, E. B. Saff, "Discretizing manifolds via minimum energy points", Notices Amer. Math. Soc., 51:10 (2004), 1186-1194. 
[23] D. P. Hardin, E. B. Saff, "Minimal Riesz energy point configurations for rectifiable d-dimensional manifolds", Adv. Math., 193:1 (2005), 174-204.

\section{C. В. Бородачов (S. V. Borodachov)}

School of Mathematics,

Georgia Institute of Technology, Atlanta, GA, USA

E-mail: borodasv@math.gatech.edu

\section{Э. Б. Сафф (E. B. Saff)}

Center for Constructive Approximation,

Department of Mathematics,

Vanderbilt University, Nashville, TN, USA

E-mail: Edward.B.Saff@Vanderbilt.Edu

\section{Д. П. Хардин (D. P. Hardin)}

Center for Constructive Approximation, Department of Mathematics, Vanderbilt University, Nashville, TN, USA

E-mail: Doug.Hardin@Vanderbilt.Edu
Поступила в редакцию

11.09.2007 и 15.02 .2008 